\title{
A D-Q Synchronous Frame Controller for Single-Phase Inverters
}

\author{
Saeed Golestan ${ }^{1}$, Mohammad Monfared ${ }^{2}$, Josep M. Guerrero ${ }^{3}$, and Mahmood Joorabian ${ }^{4}$ \\ ${ }^{1}$ Islamic Azad University-Abadan Branch, Abadan 63178-36531, Iran (Email: saeed.golestan@gmail.com) \\ ${ }^{2}$ Department of Electrical Engineering, Ferdowsi University of Mashhad, Mashhad 91775-1111, Iran (Email: m.monfared@um.ac.ir) \\ ${ }^{3}$ Department of Automatic Control Systems Computer Engineering Universitat Politècnica de Catalunya \\ ,Comte d'Urgell 187.08036 Barcelona. Spain (Email: josep.m.guerrero@upc.edu) \\ ${ }^{4}$ Department of Electrical Engineering, Shahid Chamran University, Ahvaz 61355, Iran (Email: mjoorabian@cua.ac.ir)
}

\begin{abstract}
This paper deals with design of a synchronous frame control strategy for single-phase inverter-based islanded distributed generation (DG) systems. Although, implementation of these regulators requires a minimum of two independent phases in the system, the required orthogonal phase is generated through the use of a first order all pass filter (APF). The essence of the proposed control strategy is to use a synchronous reference frame PI (SRFPI) controller to regulate output voltage, together with a simple inner capacitor current regulating loop to stabilize the system and a voltage-feedforward loop to improve the system robustness. A detailed design criterion for the proposed control strategy is presented base on a frequency-response approach. Finally, simulation results are presented to validate the proposed method.
\end{abstract}

\section{INTRODUCTION}

Most types of DG units utilize power electronic converters as the interface media with the utility grid [1]. These converters are commonly based on a Voltage Source Inverter (VSI), which can operate either in grid-connected mode or in island mode.

Grid-connected operation consists in delivering power to the local loads and to the utility grid. In such a case, the output voltage reference is often taken from the grid voltage sensing, by using a phase-locked-loop (PLL) circuit, while control system ensures that the inverter acts as a current source [2]. Currently, when the grid is not present, the inverters are normally disconnected from the grid in order to avoid islanding operation. In the coming years, inverters should be able to operate in island mode due to the high penetration of DG [3]. In addition, in certain zones where a stiff grid is not accessible, e.g., some physical islands, rural or remote areas, islanding operation mode is necessary.

Various researches on the control of the single-phase inverters in island mode have been presented in literature [4]-[7]. As low-cost, high-performance digital signal processors (DSP) have been available in recent years, applications of advanced control techniques have been reported such as the dead beat control [4], repetitive control [5], capacitor current feedback [6] and sliding-mode control [7]. The reported results have clearly shown performance improvement. However, since the control of the single-phase PWM inverter is not a regulation problem for a DC reference but a tracking problem for a sinusoidal AC reference, it is very difficult to achieve zero steady-state error.

To overcome this problem, a SRFPI controller for singlephase inverters is proposed in this paper [8]. Although the implementation of DQ regulators requires a minimum of two independent phases in the system, a second phase for implementation is constructed by applying a $90^{\circ}$ phase shift with respect to the fundamental frequency of single-phase signal. The essence of the proposed control strategy is to use a SRFPI controller to regulate the output voltage, together with a simple inner capacitor current regulating loop to stabilize the system and a voltage-feedforward loop to improve the system robustness. A detailed design criterion for the proposed control strategy is presented based on a frequency-response approach. Simulation results are reported, confirming the validity of this control technique.

\section{System Modeling}

Fig. 1 shows the converter system considered in this investigation, which includes an insulated-gate bipolar transistor (IGBT) bridge configuration driven from constant voltage DC bus and an LC filter. Note that the assumption of a constant DC voltage is reasonable if the DC capacitance is large or if DC bus voltage ripple compensation is included within the PWM control algorithm [9]. Salient parameters for the system are shown in Table I.

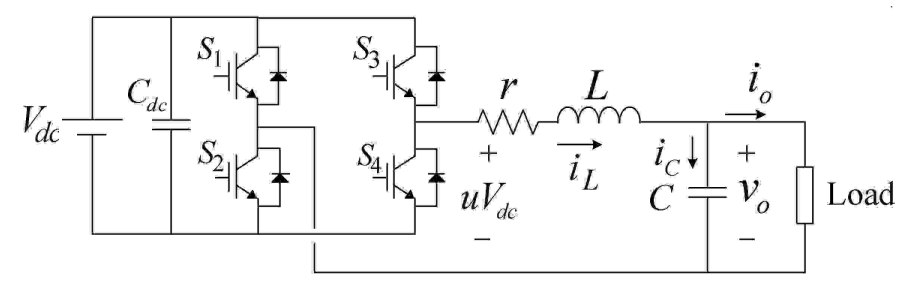

Fig. 1. Power stage of the single-phase inverter

The bilinear differential equations that describe the largesignal dynamic behavior of this converter are presented as 
TABLE I

SYSTEM PARAMETERS

\begin{tabular}{c|c}
\hline Power Stage Parameters & Nominal Value \\
\hline Switching frequency, $f_{s}$ & $20 k H z$ \\
\hline Filter inductance, $\mathrm{L}$ & $500 \mu \mathrm{H}$ \\
\hline Filter capacitance, $\mathrm{C}$ & $22 \mu \mathrm{F}$ \\
\hline ESR of the inductance, $\mathrm{r}$ & $0.2 \Omega$ \\
\hline Dc-link voltage, $V_{d c}$ & $300 \mathrm{~V}$
\end{tabular}

follows:

$$
\begin{array}{r}
L \frac{d i_{L}}{d t}=u V_{d c}-v_{o}-r i_{L} \\
C \frac{d v_{o}}{d t}=i_{C}=i_{L}-i_{o}
\end{array}
$$

where $r$ is the ESR of the inductance $L$, and $u$ is the control variable, which can take the following values: 1,0 or -1 , depending on the state of the pair of switches $S_{1}-S_{2}$ and $S_{3}-S_{4}$.

A simple time-continuous model of the inverter derived from the above equations is shown in Fig. 2, where the full-bridge is replaced by $\tilde{m} V_{d c}$, assuming that the inverter switching frequency, $f_{s}$, is much higher than the frequency of the modulating signal, $\tilde{m}$.

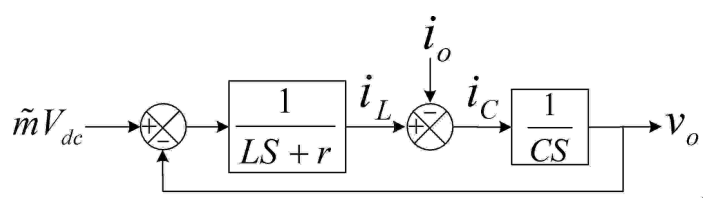

Fig. 2. A simplified model of the single phase inverter

\section{DQ Rotating Frame Concept FOR Single-Phase CONVERTERS}

Theoretically for a three phase converter, simple PI regulators designed in a DQ rotating frame, can achieve infinite loop gain ( and consequently zero steady state error ) at the fundamental frequency; However this method is not applicable to single-phase converters, since there is only one phase variable available, while the DQ transformation requires a minimum of two orthogonal variables.

In order to create the required orthogonal phase information from the original single-phase signal, different techniques can be applied [10]-[11]. In this paper, we use a first-order all-pass filter (APF), as shown in equation (3), to create the required orthogonal signal [12].

$$
A P F=\frac{w-s}{w+s}
$$

where $w$ is the fundamental frequency.

The DQ transformation can be implemented once the required orthogonal signal is generated. Equation (4) defines the transformation from stationary frame $(\alpha \beta)$ to rotating frame (DQ), and equation (5) defines the transformation from rotating frame to stationary frame.

$$
T=\left[\begin{array}{cc}
\cos (\omega t) & \sin (\omega t) \\
-\sin (\omega t) & \cos (\omega t)
\end{array}\right]
$$

$$
T^{-1}=\left[\begin{array}{cc}
\cos (\omega t) & -\sin (\omega t) \\
\sin (\omega t) & \cos (\omega t)
\end{array}\right]
$$

\section{Controller Design}

The essence of the proposed control strategy, as shown in Fig. 3, is to use a SRFPI controller to regulate the output voltage, together with a simple inner current regulating loop to stabilize the system and a voltage-feedforward loop to improve the system robustness. The feedback signal for the inner current loop can be either the filter capacitor current or the filter inductor current. Capacitor current feedback is preferred in this paper, because of its inherent ability to forecast the change of the output voltage $\left(i_{C}=C d v_{o} / d t\right)$. The capacitor current changes instantaneously with the load current change, irrespective of the inductor current, and therefore provides a rapid control action to correct the output voltage.

As illustrated in Fig. 3, the DQ components of the output voltage, $v_{o, d q}$, are first generated by applying the APF and transformation matrix given in (4). These components are then compared with reference voltages $v_{o, d q}^{*}$, and resultant errors, $E_{d q}$, are passed through two PI controllers for eliminating the tracking errors. In order to compensate coupling between $d$ and $q$ axes, decoupling terms are also included. The output signals of the voltage loop, $i_{c, d q}^{*}$, are then transferred back into the stationary frame to provide reference current for the inner current loop, where naturally only the real reference current $i_{c, \alpha}^{*}$ is fed back to compare with the measured capacitor current. The inner current loop only uses a simple proportional compensator, since it only stabilizes the control system and its steady state error do not affect the accuracy of the voltage control loop. Finally, the measured voltage $v_{o}$ is added to output of the current controller to compensate the disturbances caused by the output voltage. This compensation allows the inner current loop to have a smaller gain and hence increased robustness.

\section{A. Single-Phase Equivalent of the SRFPI regulator}

Because of the utilization of the SRFPI controller in the voltage loop, the classical control techniques can not be applied to evaluate the stability of the closed loop system. To overcome this problem, the single-phase equivalent of the SRFPI regulator is presented. This representation provides useful insights as to why a SRFPI regulator is superior to a conventional PI regulator, and significantly simplifies controller design and stability analysis [13].

The first step in the derivation of the single-phase equivalent of a SRFPI regulator is to move the PI regulator from the rotating $d q$ frame into the stationary $\alpha \beta$ frame. The equivalent $\alpha \beta$ frame representation of a SRFPI regulator is shown in Fig. 4, where $G_{P I}(s)=K_{P}+K_{i} / s$, and $E_{\alpha}$ and $E_{\beta}$ are the real and orthogonal voltage errors, respectively. Notice that decoupling terms are not considered, assuming they have a negligible effect on the performance of the control system.

Equation (6) describes time-domain $\alpha \beta$-frame representation of a SRFPI regulator, where $*$ denotes a convolution product. Expanding (6) produces (7) and (8). Each of the two outer terms in both (7) and (8) can be rearranged using 



Fig. 4. Equivalent $\alpha \beta$ frame representation of a SRFPI regulator

Fig. 3. Proposed control strategy

$$
\begin{aligned}
& {\left[\begin{array}{l}
i_{C, \alpha}^{*} \\
i_{C, \beta}^{*}
\end{array}\right]=\left[\begin{array}{cc}
\cos (\omega t) & -\sin (\omega t) \\
\sin (\omega t) & \cos (\omega t)
\end{array}\right]\left\{\left[G_{P I}(t)\right] *\left\{\left[\begin{array}{cc}
\cos (\omega t) & \sin (\omega t) \\
-\sin (\omega t) & \cos (\omega t)
\end{array}\right]\left[\begin{array}{c}
E_{\alpha} \\
E_{\beta}
\end{array}\right]\right\}\right\}} \\
& i_{C, \alpha}^{*}(t)=\left\{G_{P I}(t) *\left(\begin{array}{c}
E_{\alpha} \cdot \cos (\omega t) \\
+E_{\beta} \cdot \sin (\omega t)
\end{array}\right)\right\} \cdot \cos (\omega t)+\left\{G_{P I}(t) *\left(\begin{array}{c}
E_{\alpha} \cdot \sin (\omega t) \\
-E_{\beta} \cdot \cos (\omega t)
\end{array}\right)\right\} \cdot \sin (\omega t) \\
& i_{C, \beta}^{*}(t)=\left\{G_{P I}(t) *\left(\begin{array}{c}
E_{\alpha} \cdot \cos (\omega t) \\
+E_{\beta} \cdot \sin (\omega t)
\end{array}\right)\right\} \cdot \sin (\omega t)+\left\{G_{P I}(t) *\left(\begin{array}{c}
-E_{\alpha} \cdot \sin (\omega t) \\
E_{\beta} \cdot \cos (\omega t)
\end{array}\right)\right\} \cdot \cos (\omega t) \\
& L\left\{\left[G_{P I}(t) *\left(E_{\alpha} \cdot \cos (\omega t)+E_{\beta} \cdot \sin (\omega t)\right)\right] \cdot \cos (\omega t)\right\} \\
& =\left[G_{P I}(s) \cdot L\left\{E_{\alpha} \cdot \cos (\omega t)+E_{\beta} \cdot \sin (\omega t)\right\}\right] * \frac{s}{s^{2}+\omega^{2}} \\
& =\frac{1}{2}\left[G_{P I}(s) \cdot\left[\begin{array}{c}
E_{\alpha}(s+j \omega)+E_{\alpha}(s-j \omega) \\
+j E_{\beta}(s+j \omega)-j E_{\beta}(s-j \omega)
\end{array}\right]\right] * \frac{s}{s^{2}+\omega^{2}} \\
& =\frac{1}{4}\left[\left[\begin{array}{c}
G_{P I}(s+j \omega)\left(\begin{array}{c}
E_{\alpha}(s+2 j \omega)+E_{\alpha}(s) \\
+j E_{\beta}(s+2 j \omega)-j E_{\beta}(s)
\end{array}\right) \\
+G_{P I}(s-j \omega)\left(\begin{array}{c}
E_{\alpha}(s-2 j \omega)+E_{\alpha}(s) \\
-j E_{\beta}(s-2 j \omega)+j E_{\beta}(s)
\end{array}\right)
\end{array}\right]\right]
\end{aligned}
$$



$$
\begin{aligned}
& {\left[\begin{array}{c}
i_{C, \alpha}^{*} \\
i_{C, \beta}^{*}
\end{array}\right]=\left[\begin{array}{cc}
K_{P}+\frac{K_{i} s}{s^{2}+\omega^{2}} & -\frac{K_{i} \omega}{s^{2}+\omega^{2}} \\
\frac{K_{i} \omega}{s^{2}+\omega^{2}} & K_{P}+\frac{K_{i} s}{s^{2}+\omega^{2}}
\end{array}\right]\left[\begin{array}{c}
E_{\alpha} \\
E_{\beta}
\end{array}\right]}
\end{aligned}
$$

Laplace transforms, as shown in (9). Combining (9) and its counterparts together, and rearranging the results back into matrix form, results in (10). Note that the double frequency components shown in (9) cancel out with the other three outer terms in (7) and (8). By substituting the PI compensator transfer function, $G_{P I}(s)=K_{P}+K_{i} / s$, (10) can be further expressed as (11).

Finally, by substituting $E_{\beta}=\frac{\omega-s}{\omega+s} E_{\alpha}$ into (11), the transfer function between the real voltage error $E_{\alpha}$ and the real reference current $i_{C, \alpha}^{*}$ can be expressed as

$$
\begin{aligned}
i_{C, \alpha}^{*} & =\frac{a_{3} s^{3}+a_{2} s^{2}+a_{1} s+a_{0}}{s^{3}+\omega s^{2}+\omega^{2} s+\omega^{3}} E_{\alpha} \\
& =H(s) E_{\alpha}
\end{aligned}
$$

where $a_{3}=K_{P}, a_{2}=K_{P} \omega+K_{i}, a_{1}=K_{P} \omega^{2}+2 \omega K_{i}, a_{0}=$ $K_{P} \omega^{3}-K_{i} \omega^{2}$, and $H(s)$ is the single-phase equivalent of the SRFPI regulator, which has a frequency response characteristic as shown in Fig. 5.

Notice, the transfer function $H(s)$ has very high gain at 


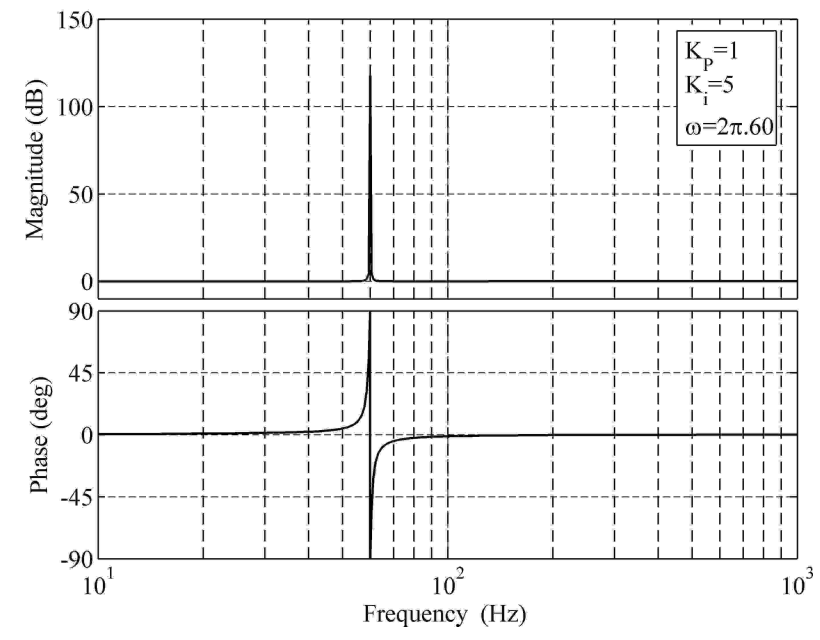

Fig. 5. Bode plot of transfer function $H(s)$

fundamental frequency. That is the reason why using a SRFPI regulator zero steady-state error at the fundamental frequency can be achieved.

\section{B. Controller Parameters Design}

The simplified model of the inverter with the added inner capacitor current control loop, is shown in Fig. 6, where the load current is represented as a disturbance input.

From Fig. 6, the closed-loop transfer function of the system is obtained as

$$
i_{C}=\frac{K}{L s+r+K} i_{C}^{*}-\frac{L s+r}{L s+r+K} i_{o}
$$

Frequency domain analysis can now be performed using (13) to plot the Bode diagrams of $i_{C} / i_{C}^{*}$ and $i_{C} / i_{o}$ as shown in Fig. 7 for the case of gain $K=1$. Ideally, the bandwidth of $i_{C} / i_{C}^{*}$ in Fig. 7 should be maximized (tend toward $\infty$ ) by using a higher $K$, to achieve perfect reference tracking at all input frequencies, a faster dynamic response and the complete blocking of disturbance input from feeding forward to the output. A high gain can however degrade the control loop stability when physically implemented. A satisfactory compromise therefore is to use a $K$ that will give a near-unity closed-loop current gain at the fundamental frequency. Based on this gain selection criterion, $K$ is set as 15 , which will give a bandwidth of $4.6 \mathrm{~K} \mathrm{~Hz}$ with a $90^{\circ}$ phase margin, a closedloop current gain of $i_{C} / i_{C}^{*}=-0.1157 \mathrm{db}=0.9868$ and a negligibly small $i_{C} / i_{o}$ gain at the fundamental frequency.

Once the inner current loop design is finished, the next step is to fine-tune the parameters of the voltage feedback loop. Fig.

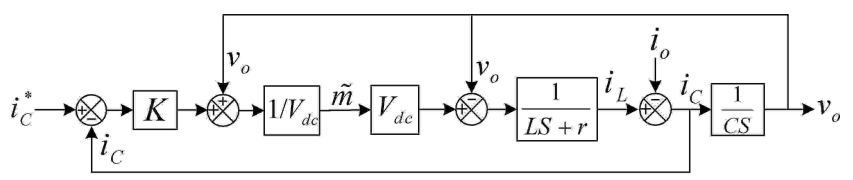

Fig. 6. Block diagram of inner current loop

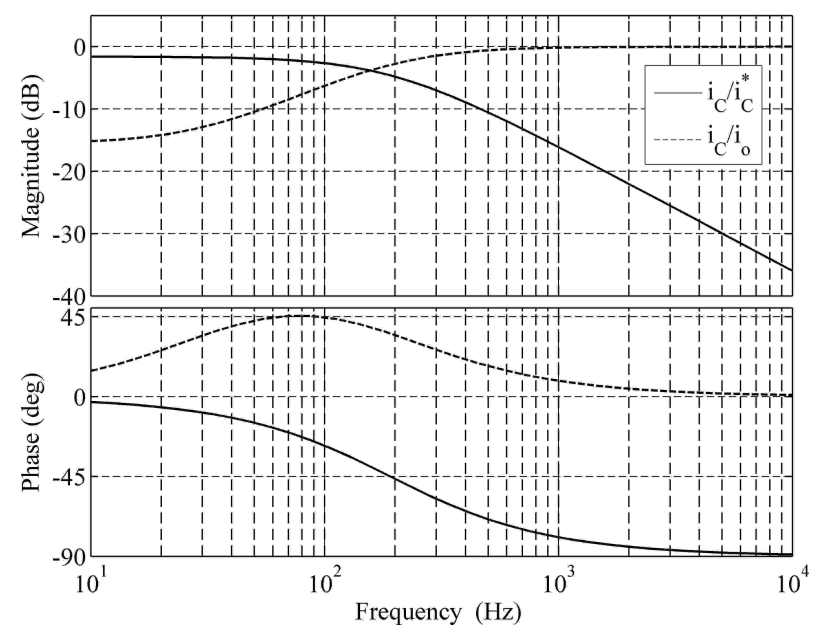

Fig. 7. Bode plots of $i_{C} / i_{C}^{*}$ and $i_{C} / i_{o}$

8 shows the single-phase equivalent of the proposed control system, in which the SRFPI regulator is replaced by $\mathrm{H}(\mathrm{s})$, and $v_{o}^{*}=v_{o, \alpha}^{*}=v_{o, d}^{*} \cos (\omega t)-v_{o, q}^{*} \sin (\omega t)$.

From Fig. 8, the open-loop and closed-loop transfer functions of the system can be written as (14) and (15), respectively, where $b=(L \omega+r+K) C$.

Analysis of closed loop system shows that its stability is primarily determined by the voltage loop proportional gain $K_{p}$, as illustrated in Fig. 9 which shows how the position of the closed loop poles of the system vary with $K_{p}$ for two different values of $K$. In both cases the system is stable for $K_{p}>K_{i} / \omega$.

The harmonic impedance is another criteria, which must be considered when determining the parameters of the voltage feedback loop (Harmonic impedance identifies the output voltage distortion caused by one unit of harmonic load current). In order to minimize the inverter harmonic voltage distortion under distorted load conditions, the harmonic impedance should be ideally zero.

From Fig. 8, the transfer function of the harmonic impedance can be derived as (16), where $d=L \omega+r$.

Fig. 10 shows the harmonic impedance magnitude and phase verses frequency for three values of $K_{p}: K_{p}=0.5$ (solid line), $K_{p}=1$ (dashed line) and $K_{p}=1.5$ (dotted line). It can be seen from this figure that while the harmonic impedance goes through a maximum close to the LC filter resonant frequency, $f_{r e s}$, its absolute magnitude decreases as $K_{p}$ increases. Hence the inverter harmonic voltage distortion under distorted load conditions will decrease for a higher proportional gain $K_{p}$.

Based on this results, a value of $K_{p}=0.8$ is chosen to achieve a good transient response, together with an acceptable phase margin, and a relatively low impedance at harmonic frequencies.

The integral gain, $K_{i}$, of the controller acts to eliminate steady state error at the fundamental frequency. Due to the complexity of the plant transfer function, simulation techniques has been used to tune this value. The integral gain 


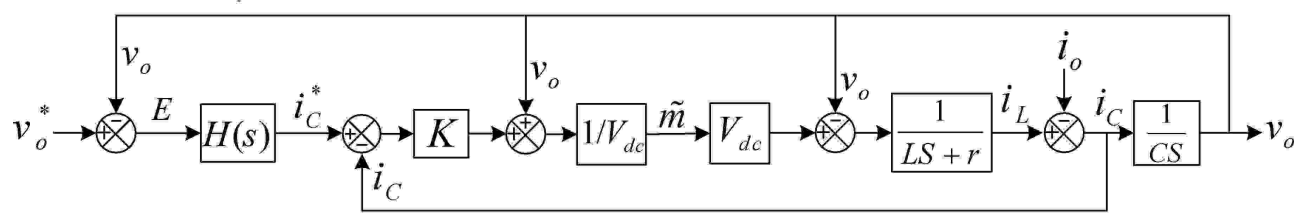

Fig. 8. Single-phase equivalent of the control system,

$$
\begin{gathered}
G_{o l}(s)=\frac{v_{o}}{v_{o}^{*}-v_{o}}=\frac{K\left(a_{3} s^{3}+a_{2} s^{2}+a_{1} s+a_{0}\right)}{L C s^{5}+b s^{4}+b \omega s^{3}+b \omega^{2} s^{2}+(r+K) C \omega^{3} s} \\
G_{C l}(s)=\frac{v_{o}}{v_{o}^{*}}=\frac{G_{o l}(s)}{G_{o l}(s)+1}=\frac{K\left(a_{3} s^{3}+a_{2} s^{2}+a_{1} s+a_{0}\right)}{L C s^{5}+b s^{4}+\left(b \omega+K a_{3}\right) s^{3}+\left(b \omega^{2}+K a_{2}\right) s^{2}+\left((r+K) C \omega^{3}+K a_{1}\right) s+K a_{0}}
\end{gathered}
$$

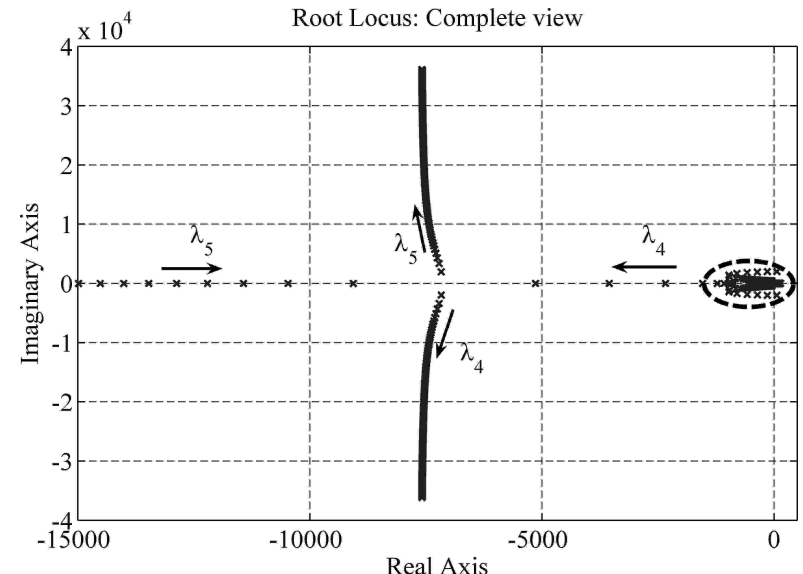

(a)



(c)

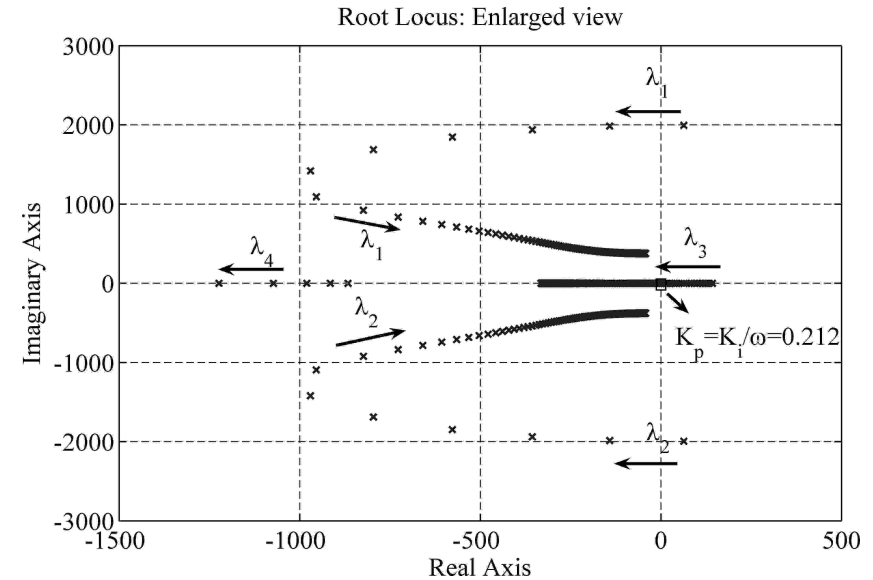

(b)

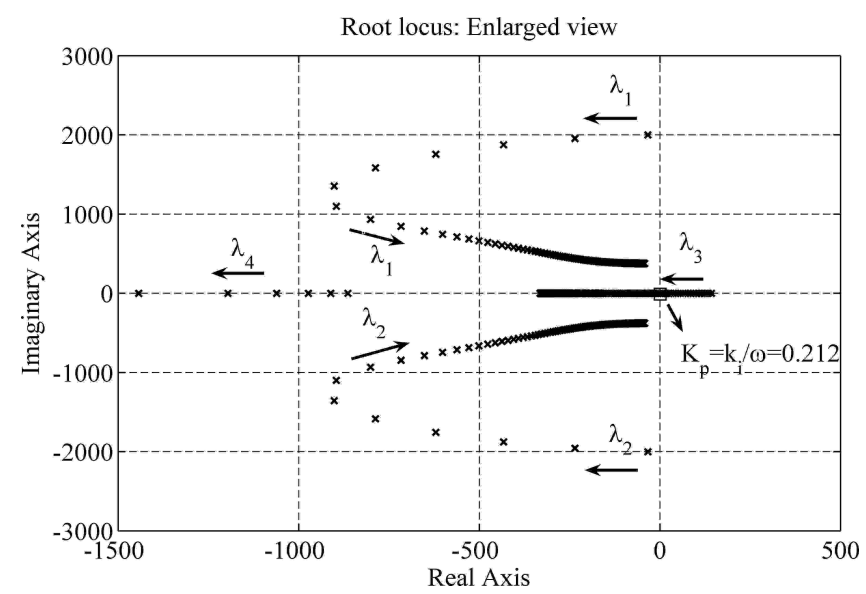

(d)

Fig. 9. Variation of closed loop poles of the system with respect to $K_{p}$. (a) and (b): $0<K_{P}<2, K_{i}=80, K=15$. (c) and (d): $0<K_{P}<2, K_{i}=$ $80, K=80$.

$$
Z_{o}(s)=\frac{v_{o}}{i_{o}}=\frac{L s^{4}+d s^{3}+d \omega s^{2}+d \omega^{2} s+r \omega^{3}}{L C s^{5}+b s^{4}+\left(b \omega+K a_{3}\right) s^{3}+\left(b \omega^{2}+K a_{2}\right) s^{2}+\left((r+K) C \omega^{3}+K a_{1}\right) s+K a_{0}}
$$






Fig. 10. Variation of the harmonic impedance with respect to $K_{p}$. (arrow direction indicates increasing value of $K_{p}$ )

required to achieve a desired steady-state phase and amplitude error is found in this way to be $K_{i}=80$.

\section{Simulation Results}

The performance of the proposed control strategy has been investigated using MATLAB/SIMULINK simulations. To better approximate a physical system, all simulation investigations were performed with digital sampling effects, inverter switching, and measurement and computational delays incorporated. In all the simulation cases, the nominal frequency is $60 \mathrm{~Hz}$; the nominal voltage is $120 \sqrt{2} \mathrm{~V}$; the sampling frequency is $40 \mathrm{KHz}$, and nominal power of the inverter is $2 \mathrm{kVA}$.

Fig. 11 shows the time responses of the output voltage $v_{o}(t)$ together with the output voltage reference $v_{o}^{*}$ (left plot), and the tracking error $E(t)$ (right plot), using the proposed controller under the rated resistive load. Notice that the output voltage $v_{o}(t)$ is almost a sinusoidal signal and has an excellent tracking over its reference $v_{o}^{*}$, and thus the tracking error $E(t)$ is made relatively small (less than 3 Vpeak in steady-state). In order to compare the performance of the proposed controller, we have also implemented a conventional multiloop PI control, designed with the same bandwidth and the same phase margin. Fig. 12 shows the results obtained with the same resistive load used for Fig. 11, highlighting the advantages of the proposed controller.

To verify transient performance, the behavior of the converter under a triac controlled load (load is changed from no load to nominal load) with a firing Angle of $90^{\circ}$ every half cycle is investigated. The performance of the controller, is very good, as shown in Fig. 13, the load transient is recovered in around $0.2 \mathrm{~ms}$, and the THD is limited to $2.24 \%$.

The proposed controller has been evaluated in the presence of a distorting load (diode rectifier load followed by a $700 \mu \mathrm{F}$ capacitor in parallel with a $30 \Omega$ resistor). Fig. 14 reports the results of the proposed controller showing that the output voltage waveform exhibits small deviations from the ideal sinusoidal waveform and the THD is limited to $1.97 \%$.

\section{CONCLUSION}

A synchronous reference frame PI (SRFPI) controller has been presented to regulate the instantaneous output voltage of the single phase inverter with superior dynamic response as well as zero steady-state error at the fundamental frequency. The essence of the proposed controller is to use a SRFPI controller to regulate the output voltage, together with a simple inner capacitor current regulating loop to stabilize the system and a voltage-feedforward loop to improve the system robustness. Because of the use of the SRFPI controller in the voltage loop, the classical control techniques can not be applied to evaluate the stability of the closed loop system. Thus, the single-phase equivalent of the SRFPI regulator was provided, which, significantly simplifies controller design and stability analysis. A detailed design procedure with consideration of the practical implementation aspects has been presented and applied to the current and voltage control loops. The performance of the proposed control strategy has been investigated using MATLAB/SIMULINK simulations. The simulation results exhibit satisfactory steady state and transient performance, particularly under nonlinear loads.

\section{REFERENCES}

[1] F. Gao and M. R. Iravani, "A control strategy for a distributed generation unit in grid-connected and autonomous modes of operation," IEEE Trans. Power Del., vol. 23, no. 2, pp. 850-859, Apr. 2008.

[2] J. M. Guerrero, J. Matas, L. García de Vicuña, M. Castilla, and J. Miret, "Wireless-control strategy for parallel operation of distributed-generation inverters," IEEE Trans. Ind. Electron., vol. 53, no. 5, pp. 14611470, Oct. 2006.

[3] P. L. Villeneuve, "Concerns generated by islanding," IEEE Power Energy Mag., vol. 2, no. 3, pp. 49-53, May/Jun. 2004.

[4] P. Mattavelli, "An improved dead-beat control for UPS using disturbance observers," IEEE Trans. Ind. Electron., Vol. 52, No. 1, pp. 206212, Feb. 2005.

[5] Y. Y. Tzou, R. S. Ou, S. L. Jung, M. Y. Chnag, ”High-performance programmable AC power source with low harmonic distortion using DSPbased repetitive control technique", IEEE Trans. Power Elec., Vol. 12, pp. 715-725, July 1997.

[6] N. M. Abdel-Rahim and J. E. Quaicoe, "Analysis and design of a multiple feedback loop control strategy for single-phase voltage-source UPS inverters," IEEE Trans. Power Elec., vol. 11, pp. 532-541, July 1996.

[7] S. L. Jung, and Y. Y. Tzou, "Discrete sliding-mode control of a PWM inverter for sinusoidal output waveform synthesis with optimal sliding curve", IEEE Trans. Power Electron., 1996, pp. 567577.

[8] A. Roshan, R. Burgos, A. Baisden, F. Wang, D. Boroyevich, "A D-Q frame controller for a full-bridge single phase inverter used in small distributed power generation (DG) systems," in Proc. IEEE APEC, 2007, pp. 641-647.

[9] E. Twining and D. G. Holmes, "Grid Current Regulation of a Three-Phase Voltage Source Inverter With an LCL Input Filter," IEEE Trans. Power Electro., Vol. 18, No. 3, pp .888-895, , may 2003.

[10] S. Sakamoto, T. Izumi, T. Yokoyama, and T. Haneyoshi, "A new method for digital PLL control using estimated quadrature two phase frequency detection," in Proc. PCC, Apr. 2002, pp. 671676.

[11] M. Saitou, N. Matsui, T. Shimizu, "A control strategy of single-phase active filter using a novel d-q transformation," in Proc. IEEE IAS, 2003, pp. 1222-1227.

[12] B. H. Kwon, J. H. Choi, and T. W. Kim, "Improved single-phase lineinteractive UPS," IEEE Trans. Ind. Electron., vol. 48, no. 4, pp. 804-811, Aug. 2001.

[13] D. N. Zmood and D. G. Holmes, Stationary frame current regulation of PWM Inverters with zero-steady-state error, IEEE Trans. Power Electr., vol.18, no.3, pp. 814-822, May 2003. 


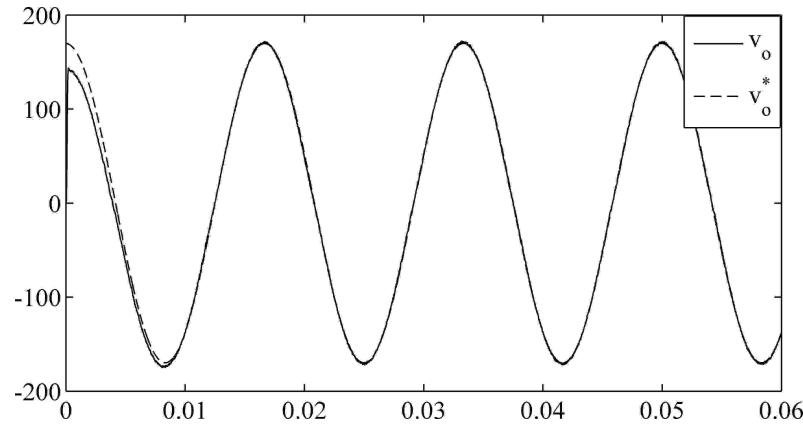

(a)

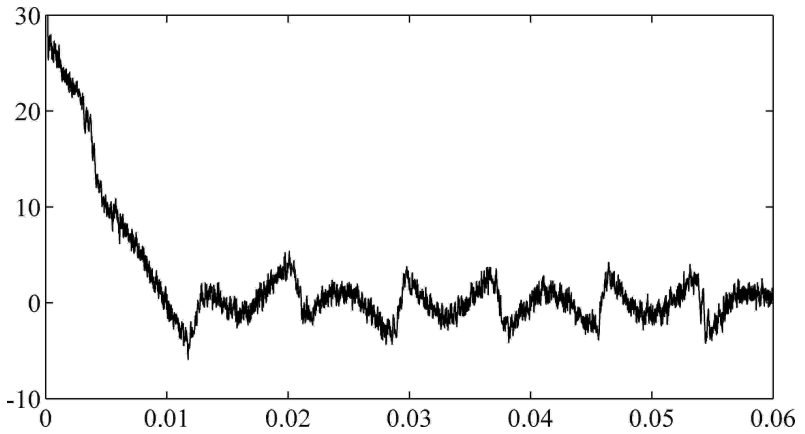

(b)

Fig. 11. Simulation results for the proposed controller under a resistive load. (a): output voltage $v_{o}$ (solid line) together with output voltage reference $v_{O}^{*}$ (dashed line), (b): tracking error $E(t)$.

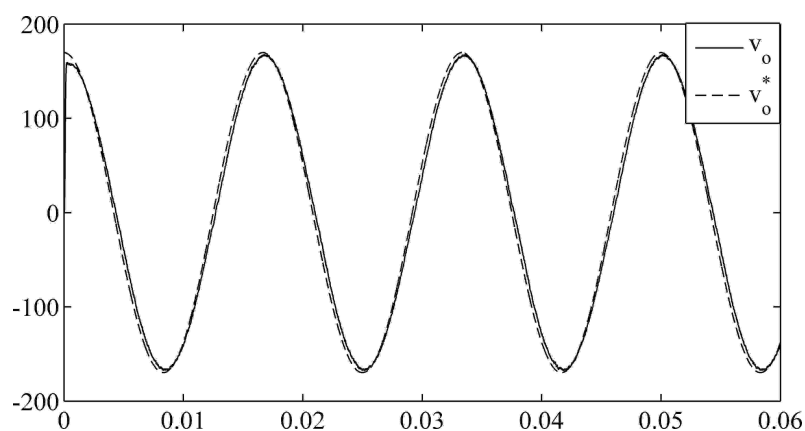

(a)



(b)

Fig. 12. Simulation results for the multiloop PI controller under a resistive load. (a): output voltage $v_{o}$ (solid line) together with output voltage reference $v_{o}^{*}$ (dashed line), (b): tracking error $E(t)$.



(a)

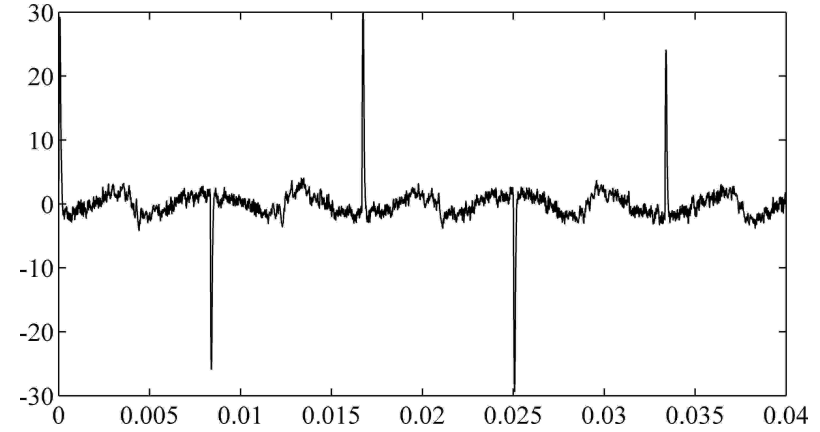

(b)

Fig. 13. Simulation results for the proposed controller under a triac controlled load. (a): output voltage $v_{o}$ and load current $i_{O}$, (b): tracking error $E(t)$ harmonics spectrum.

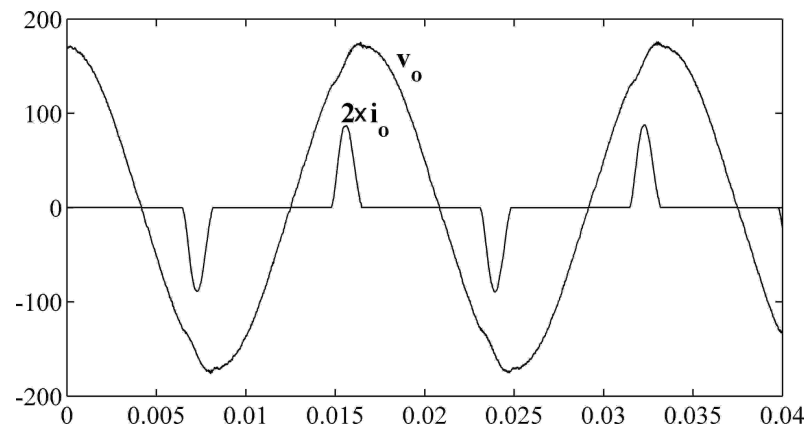

(a)

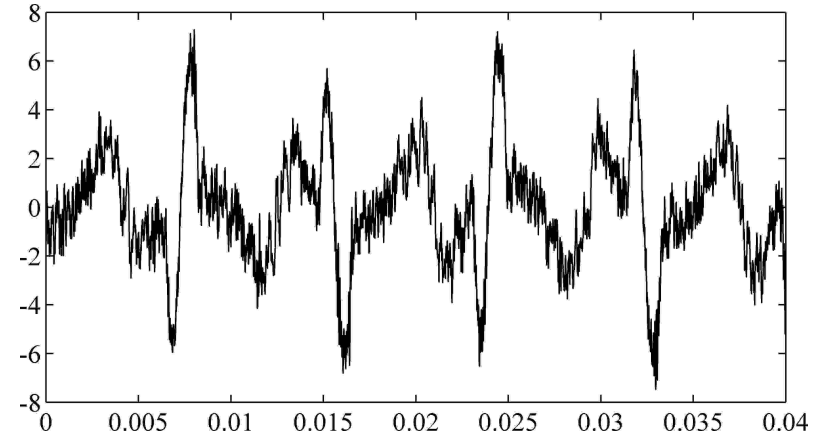

(b)

Fig. 14. Simulation results for the proposed controller under a rectifier load. (a): output voltage $v_{O}$ and load current $i_{o}$, (b): tracking error $E(t)$ harmonics spectrum 